\title{
Gastric Ulcer Associated with Cytomegalovirus in an Immunocompetent Patient: Method for Diagnosis
}

\author{
Chikara Ebisutani $^{a} \quad$ Akira Kawamura $^{a}$ Norihito Shibata $^{\mathrm{a}}$ \\ Masamichi Nasu ${ }^{a}$ Rei Ueno ${ }^{a}$ Keiko Mimura ${ }^{b}$ \\ Yoshikazu Kinoshita ${ }^{\mathrm{C}}$ \\ Departments of a Gastroenterology and ${ }^{b}$ Clinical Laboratory, Pathology Section, \\ Ono Municipal Hospital, Ono, and ${ }^{\mathrm{C}}$ Department of Gastroenterology and \\ Hepatology, Shimane University School of Medicine, Izumo, Japan
}

\section{Key Words}

Cytomegalovirus $\cdot$ Stomach ulcer $\cdot$ Immunohistochemistry $\cdot$ Endoscopy

\begin{abstract}
Cytomegalovirus (CMV)-associated gastric ulcers can be found not only in immunocompromised hosts but also in normal individuals. The accurate endoscopic diagnosis of CMV ulcers is not easy because of the absence of characteristic morphological features. We present a case of CMV-associated gastric ulcer in an immunocompetent patient. He was a 33-year-old male with epigastralgia. Upper gastrointestinal endoscopy showed multiple erupted papules and a large irregularly shaped shallow ulcer. We did not find intracellular inclusion bodies characteristic of CMV in hematoxylin-eosin-stained gastric biopsy specimens, while CMV antigens corresponding to intracellular inclusion bodies were confirmed using an immunoperoxidase method with the monoclonal CMV antibody. If CMV ulcers are suspected, it is important to examine for inclusion bodies using not only hematoxylin-eosin staining, but also CMV immunohistochemistry for a sensitive diagnosis.
\end{abstract}

\section{Introduction}

Infection by cytomegalovirus (CMV) is not a rare disease and is frequently observed in immunocompromised hosts with hematological/immunological diseases or under treatment with glucocorticoids or immunosuppressants. The majority of patients with CMV infection are asymptomatic or minimally symptomatic, while CMV-associated disease can easily become severe in immunocompromised hosts [1]. Although CMV 
infection occurs in any organ, the gastrointestinal tract is one of the organs where it frequently occurs. In patients with ulcerative colitis for example, CMV infection, which is frequently observed in inflamed colon, is a well-known aggravating factor of disease activity and patient prognosis [2]. CMV infection in the colon and small intestine usually causes shallow erosions and erythemas without characteristic morphological features useful for diagnosis. CMV infection in the stomach is frequently reported in normal immunocompetent hosts and is reported to cause gastric ulcers that are difficult to differentiate from Helicobacter pylori- or non-steroidal anti-inflammatory drug (NSAID)-related ulcers and are often accompanied by systemic inflammatory responses such as fever. Even in these cases, the diagnosis of CMV gastric ulcer is not easy, partly because of the absence of characteristic morphological features in gastric ulcers and partly because of the difficulty in the identification of CMV infection in biopsy specimens by routine histomorphological examinations [3-5]. Therefore, in cases with gastric ulcer and possible CMV infection, additional tests need to be added as appropriate. Immunohistological detection of CMV antigens, in situ hybridization to detect CMV DNA, and PCR amplification detection of CMV DNA may be the possible additions. We experienced an immunocompetent patient with possible CMV gastric ulcers and made an appropriate and prompt diagnosis with the help of immunohistological examination. We show the case and emphasize the value of immunohistological testing for the diagnosis of CMV gastric ulcers.

\section{Case Report}

A 33-year-old man came to our hospital in July 2011 with epigastralgia. A cough and low-grade fever had been present for 3 weeks, while epigastralgia had not developed until 2 days prior. There was no other history of prior illness, surgery or blood transfusion. A blood examination revealed mild liver damage and laboratory data showed the following: white blood cells 9,240 cells $/ \mathrm{mm}^{3}$, neutrophils $34.7 \%$, monocytes $6.6 \%$, lymphocytes $52.8 \%$, eosinophils $4.2 \%$ and basophils $1.7 \%$. Atypical lymphocytes were not found. In addition, aspartate aminotransferase was at $51 \mathrm{IU} / \mathrm{l}$, alanine aminotransferase $87 \mathrm{IU} / \mathrm{l}$, lactate dehydrogenase $304 \mathrm{IU} / \mathrm{l}$, gamma-glutamyl transpeptidase $66 \mathrm{IU} / \mathrm{l}$, alkaline phosphatase $280 \mathrm{IU} / \mathrm{l}$ and total bilirubin $0.70 \mathrm{mg} / \mathrm{dl}$. Serological tests indicated no recent infection with hepatitis $B$, hepatitis $C$, human immunodeficiency virus or syphilis, while seropositive findings were noted for CMV-IgM, CMV-IgG and H. pylori antibodies.

The patient underwent an upper gastrointestinal endoscopy examination which revealed multiple erupted papules and a large irregularly shaped shallow ulcer (fig. 1). Multiple shallow ulcers were reported in CMV ulcer [5], but multiple erupted papules were rare and suggested infectious disease. At first we did not find intracellular inclusion bodies, a characteristic of CMV, in hematoxylin-eosin-stained gastric biopsy specimens taken from the papules and ulcer. However, added immunohistochemical analysis revealed the presence of CMV antigens in the nuclei of epithelial cells. Inclusion bodies were confirmed on day 0, but not on days 40 or 68 .

Epigastric pain disappeared soon after administration of the proton pump inhibitor (PPI) rabeprazole at $20 \mathrm{mg}$ /day. Follow-up endoscopy examinations were performed on days 40 and 68 after the start of treatment. The findings on day 40 showed a small gastric ulcer with no evidence of papules, while those on day 68 showed only a residual ulcer scar.

\section{Discussion}

Diagnosis of CMV gastric ulcers is not always easy, as their macromorphological characteristics are only slightly different from those of $\mathrm{H}$. pylori- or NSAID-related 
ulcers. Since CMV infections are not rare in immunocompromised hosts, a diagnosis of CMV ulcer is rather easy to obtain, whereas that in immunocompetent hosts is much more difficult because of its rarity. In the present case, we noted low-grade fever, peripheral blood lymphocytosis and an irregularly shaped gastric ulcer accompanied by multiple erosions without proven administration of NSAIDs and/or aspirin [4, 5]. Thus, clinical suspicion of a CMV ulcer should be raised when the above-mentioned characteristics are found in patients with gastric ulcers.

An endoscopic biopsy of the ulcer edge to determine the presence of nuclear inclusion bodies is a standard examination for confirming a diagnosis of CMV ulcer. However, identification of nuclear inclusion bodies in routine hematoxylin-eosinstained sections has been reported to be difficult and not sensitive for detection of CMV infection [6]. If we suspect CMV ulcers, we should find inclusion bodies with CMV immunohistochemistry. In addition to histological identification of nuclear inclusion bodies, detection of CMV protein or nucleic acid by immunohistochemistry or by in situ hybridization is also recommended.

In our patient, administration of a PPI for acid suppression was adequate to heal the ulcer without concomitant administration of an antiviral agent such as ganciclovir. The speed of healing in our case was considered to be not delayed in comparison with H. pylori-related ulcers, since the present ulcer healed within 10 weeks. Since the majority of H. pylori-related ulcers heal within 8 weeks [7], PPI administration may be useful for facilitating CMV ulcer healing in immunocompetent hosts, though additional studies are necessary to investigate the use of PPI therapy.

In conclusion, we treated an immunocompetent host with a CMV gastric ulcer and related erosions. We also noted the value of immunohistochemical detection of the CMV antibody for diagnosis as well as the usefulness of PPI administration for treatment of CMV gastric ulcers. 

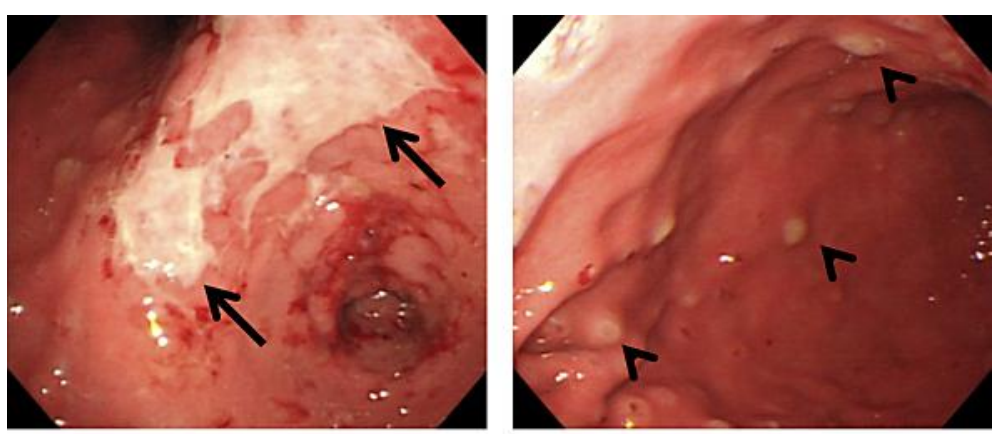

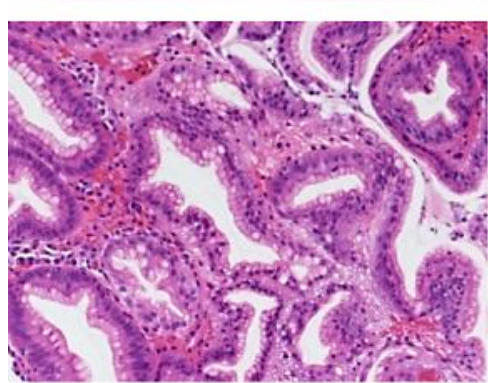

HE stain

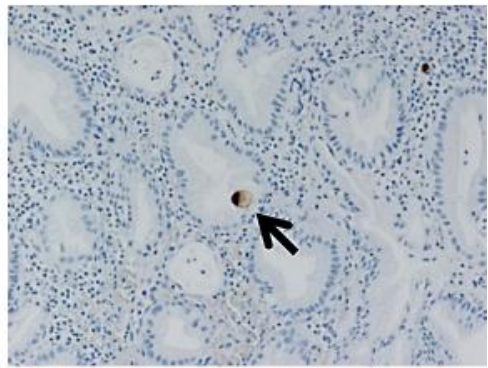

CMV immunostain

Fig. 1. Endoscopic (upper panels) and histological (lower panels: left, hematoxylin-eosin staining; right, immunostaining with anti-CMV antibody) findings of the gastric lesion when the diagnosis was made. Endoscopy revealed multiple erupted papules (arrowheads) and a large irregularly shaped shallow ulcer (long arrows). An inclusion body (short arrow) was found in the immunostained but not in the hematoxylin-eosin-stained samples. Original magnification: $\times 100$.

\section{References}

$>1$ Cohen JI, Corey GR: Cytomegalovirus infection in the normal host. Medicine 1985;64:100-114.

2 Nguyen M, Bradford K, Zhang X, Shih DQ: Cytomegalovirus reaction in ulcerative colitis patients. Ulcers DOI: $10.1155 / 2011 / 282507$.

-3 Allen JI, Silvis SE, Sumner HW, McClain CJ: Cytomegalic inclusion disease diagnosed endoscopically. Dig Dis Sci 1981;26:133-135.

-4 Himoto T, Goda F, Okuyama H, Kono T, Yamagami A, Inukai M, Masugata H, Kobayashi M, Inoue H, Kinekawa F, Masaki T, Haba R, Ohashi E, Mori T, Senda S: Cytomegalovirus-associated acute gastric mucosal lesion in an immunocompetent host. Intern Med 2009;48:1521-1524.

-5 Péter A, Telkes G, Varga M, Sárváry E, Kovalszky I: Endoscopic diagnosis of cytomegalovirus infection of upper gastrointestinal tract in solid organ transplant recipients: Hungarian single-center experience. Clin Transplant 2004;18:580-584.

6 de Castro ML, Tardío A, del Campo V, Estévez A, Pineda JR, Domínguez F, Hermo JA, Clofent J: A comparative study of two histological techniques for the identification of cytomegalovirus infection in colorectal biopsies from patients with chronic inflammatory bowel disease. Rev Esp Enferm Dig 2009;101:697-705.

-7 Higuchi K, Fujiwara Y, Tominaga K, Watanabe T, Shiba M, Nakamura S, Oshitani N, Matsumoto T, Arakawa T: Is eradication sufficient to heal gastric ulcers in patients infected with Helicobacter pylori? A randomized, controlled, prospective study. Aliment Pharmacol Ther 2003;17:111-117. 